\title{
A theoretical comparison between interferometric and optical beam deflection technique for the measurement of cantilever displacement in AFM
}

\author{
Constant A.J. Putman *, Bart G. de Grooth, Niek F. van Hulst and Jan Greve \\ Department of Applied Physics, University of Twente, P.O. Box 217, 7500 AE Enschede, The Netherlands
}

Received 12 August 1991

\begin{abstract}
A shot-noise- and diffraction-limited Michelson interferometer and two optical beam deflection configurations are compared for application in an atomic force microscope. The results show that under optimal conditions the optical beam deflection method is just as sensitive as the interferometer. This remarkable result is explained by indicating the physical equivalence of both methods.
\end{abstract}

\section{Introduction}

In the original set-up of the atomic force microscope of Binnig et al. [1], the cantilever displacement was measured by detecting changes in tunneling current between a tunnel tip and the backside of the cantilever. In the past few years a variety of other methods to measure the cantilever displacement have been described including capacitance measurement $[2,3]$ and various optical methods. For a detailed analysis of the sensitivity of the capacitance method we refer to ref. [3]. The optical methods appear to be the most used and can be divided into two categories: interferometry [4-9] and optical beam deflection (OBD) $[10,11]$. The OBD method is successfully used in photothermal deflection spectroscopy; a description of the physical background of this technique can be found in ref. [12].

Intuitively one would expect that interferometry is more sensitive than optical beam deflection. In practice, however, atomic resolution is obtained equally well with both systems, whereas an optical beam deflectometer is much simpler to

* To whom correspondence should be directed. construct than an interferometer. This has led us to take a closer look at the theoretical sensitivity of both methods. We will show that in terms of signal-to-noise ratio both principles yield very similar results. In fact, from a physical point of view, both methods are equivalent.

\section{The sensitivity of an ideal Michelson interfer- ometer}

We will first find an expression for the signalto-noise ratio of an ideal interferometer. Fundamentally the minimal displacement that can be measured with an interferometer is determined by the wave nature of light and the Poisson disitribution of the photon-emission rate of the light source. Other sources of noise, such as beam-pointing instabilities, light power fluctuations due to power supply or thermal fluctuations, are of a less fundamental nature and will be disregarded here.

We consider the basic set-up of an ideal Michelson interferometer where the measuring beam is reflected by the backside of the cantilever of an AFM (fig. 1). For simplicity the interferometer uses a collimated laser beam which 


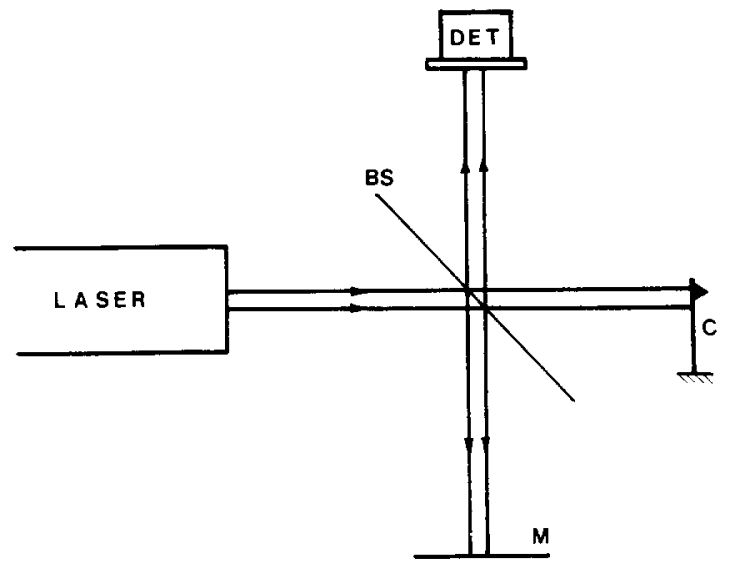

Fig. 1. Set-up of a Michelson interferometer. The object beam incident on the cantilever $\mathrm{C}$ interferes at the detector DET with the reference beam from the mirro $M$. BS is a beam splitter.

is completely reflected by the cantilever. In practice the laser beam is focused on the cantilever since the size of the reflecting surface of the cantilever is usually smaller than the collimated laser beam. This is, however, not essential for our analysis.

If the photon-emission rate of the laser is $N_{\text {tot }} / \mathrm{s}$ the detector will detect between 0 and $N_{\text {tot }}$ photons (per second), when the cantilever position varies over a distance of $\lambda / 2$ around its equilibrium position. The sensitivity of the interferometer is highest when the slope of the curve in fig. 2 is maximal, e.g. at point $\mathrm{Q}$ (operation at quadrature). A small cantilever displacement $\Delta z$

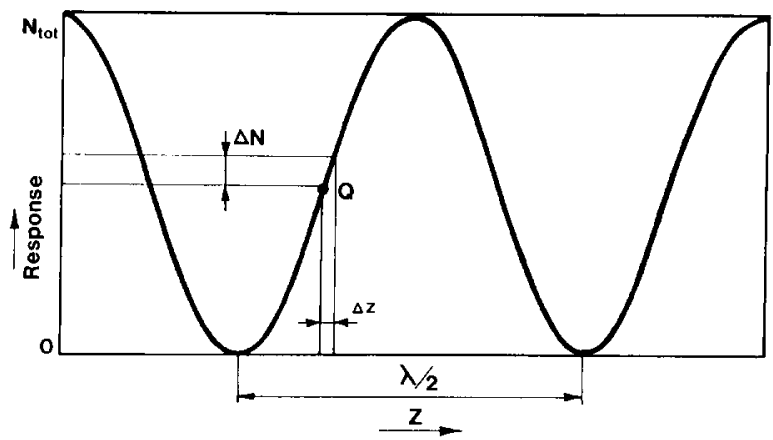

Fig. 2. Response of the interferometer as a function of the tip position, $z$. At quadrature, point $Q$, a change in cantilever position $\Delta z$, causes a change in the amount of photons incident on the detector, $\Delta N$. results in a change in the number of photons incident at the detector of

$\Delta N=(2 \pi / \lambda) t N_{\text {tot }} \Delta z$,

where $\lambda$ is the wavelength of the laser light and $t$ is the measuring time. The uncertainty in the measurement of $\Delta N$ is determined by the statistical variation of the total number of photons detected. The value of the equilibrium state has been obtained over a long period of time in order to minimize the uncertainty of that signal. For $\Delta N \ll N_{\text {tot }}$ we can write for the signal-to-noise ratio

$$
\begin{aligned}
\mathrm{SNR}_{\text {inf }} & =\Delta N /\left(t N_{\mathrm{tot}} / 2\right)^{1 / 2} \\
& =\left(8 \pi^{2} t N_{\mathrm{tot}}\right)^{1 / 2} \Delta z / \lambda,
\end{aligned}
$$

where it is assumed that an ideal photon-counting detector with a quantum efficiency of 1 and negligible dark-count rate is used. Adaptation of the formula for realistic detectors is straightforward but not necessary for a comparison with the optical beam deflection method.

\section{The sensitivity of an ideal optical beam deflec- tion system}

In analyzing the fundamental sensitivity of the optical beam deflection method it is clarifying to

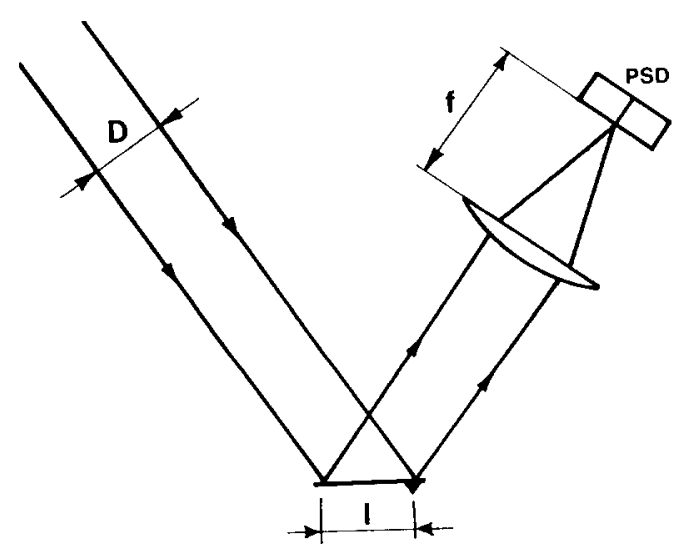

Fig. 3. Collimated laser beam (diameter $D$ ) incident on the cantilever (length $l$ ). The reflected light is focused by a lens (focal length $f$ ) onto the position-sensitive detector (PSD). 
consider first the set-up of fig. 3. A collimated Gaussian laser beam of diameter $D$ is totally reflected by the cantilever of length $l$ and focused by a lens (focal length $f$ ) on the center of a split detector. For simplicity it is assumed that the angle between laser beam and normal to the cantilever is small. In the focal plane of the lens the irradiance distribution is still Gaussian with a waist diameter given by

$d_{0}=4 \lambda f / \pi D$.

A cantilever displacement $\Delta z$ results in a shift of the laser spot at the split detector $\Delta s$

$\Delta s=2 f \Delta z / l$,

where it is assumed that the cantilever is displaced as a solid body and the separation of the two detectors is much smaller than $d_{0}$. The displacement of the laser spot is measured by subtracting the signals of the two detectors of the split detector. Assuming ideal photon counters as detectors we can write for this signal

$\Delta N=t N_{\text {tot }} \times 2 \times(2 \pi)^{1 / 2}(D / l)(\Delta z / \lambda)$.

The numerical factor is due to the Gaussian shape of the irradiance distribution at the split detector. The uncertainty in the difference signal is again determined by the statistical variation in the number of photons that are detected by the two detectors during the measurement. Assuming a Poisson distribution for the photon-emission rate, we have for the signal-to-noise ratio

$$
\begin{aligned}
\mathrm{SNR}_{\mathrm{obd} 1} & =\Delta N /\left(t N_{\mathrm{tot}} / 2+t N_{\mathrm{tot}} / 2\right)^{1 / 2} \\
& =\left(8 \pi t N_{\mathrm{tot}}\right)^{1 / 2}(D / l)(\Delta z / \lambda) .
\end{aligned}
$$

Note that the expression is not dependent on the focal length of the lens. The equation is in agreement with the proportionality relation stated by Meyer and Amer [10]. The relation suggests that $D / l$ should be made as large as possible. This ratio is limited to a value of about one by the requirement in our analysis that all the laser light is reflected by the cantilever. If the diameter of the laser $D$ exceeds the length of the cantilever, light will be lost and the irradiation pattern in the

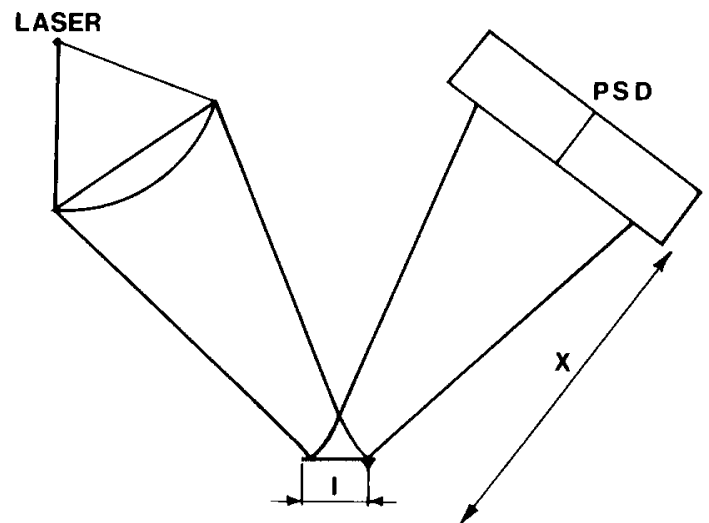

Fig. 4. Laser beam (from a diode laser) focused by a lens at the cantilever. The reflected beam is detected by the PSD. Distance between cantilever and PSD is $X$.

focal plane of the lens will no longer be Gaussian. Both effects can be shown to decrease the signalto-noise ratio. In the optimal case for which $D=l$, the expression for the signal-to-noise ratio of the OBD method reduces to

$\mathrm{SNR}_{\text {obd } 1}=\left(8 \pi t N_{\text {tot }}\right)^{1 / 2} \Delta z / \lambda$.

If we compare this with the expression obtained for the Michelson interferometer, eq. (2), we obtain the remarkable result that both methods yield essentially the same sensitivity.

The set-up discussed above cannot be used in a real AFM because the length of most cantilevers is so small that due to diffraction a collimated laser beam of this size cannot be constructed. Therefore, we consider the situation of fig. 4 where the Gaussian laser beam is focused onto the cantilever. Let the waist at the cantilever be $D_{0}$. After reflection the diameter of the beam at a distance $x$ from the cantilever is given by [13]

$D(x)=D_{0}\left[1+\left(4 \lambda x / \pi D_{0}^{2}\right)^{2}\right]^{1 / 2}$.

The ideal split detector is now positioned at a distance $x$ from the cantilever and centered in the beam. The difference signal for a small displacement $\Delta z$ of the cantilever is given by

$\Delta N=8(2 / \pi)^{1 / 2} t N_{\text {tot }}(x / D(x))(\Delta z / l)$. 
This yields a signal-to-noise ratio of

$$
\begin{aligned}
\operatorname{SNR}_{\mathrm{obd} 2}= & 8\left(t N_{\mathrm{tot}} \times 2 / \pi\right)^{1 / 2} \\
& \times \frac{x}{D_{0}\left[1+\left(4 \lambda x / \pi D_{0}^{2}\right)^{2}\right]^{1 / 2}} \Delta z / l .
\end{aligned}
$$

We see that the signal-to-noise ratio in this situation is maximal when $x \gg \pi D_{0}^{2} / 4 \lambda$. In that case the formula is exactly the same as in the previous case, eq. (6). That means that when $D_{0} / l$ is close to the maximal allowed value of one, also this configuration of the optical beam deflection method is just as sensitive as the interferometric method.

\section{Discussion}

We have found that the sensitivity of a Michelson interferometer and that of two versions of the optical beam deflection method for the measurement of cantilever displacements are essentially the same. This suggests that the fundamental physical principles underlying both methods are also the same. In the following discussion we will show this by noticing that a beam displacement method can be viewed as an interferometric technique as well.

Suppose the collimated laser beam in the scheme of fig. 3 is replaced by two parallel collimated beams originating from the same coherent light source. The first beam is reflected by the tip of the cantilever, the second beam from a position close to the base of the cantilever. In the focal plane of the lens the two beams completely overlap and form an interference pattern at the detector surface. A displacement of the tip of the cantilever results in a shift of this interference pattern. By a measurement of this shift we have constructed an interferometric method to detect the cantilever displacement. In fact, the interferometer described by Den Boef [7] is very similar to this. The sensitivity of this interferometric method is equivalent to any other ideal interferometer such as the Michelson interferometer described in fig. 1. The sensitivity of an interferome- ter is optimal when two conditions are fulfilled. Firstly, the two beams must overlap completely. In that case all information on the phase difference between the two beams iscontained in the interference pattern. In the hypothetical set-up described above this is achieved in the focal plane of the lens. Secondly, the change in phase difference between the two beams due to a displacement of the cantilever should be maximal. This condition is also fulfilled since one beam is at the tip of the cantilever and the other at the base.

In the OBD set-up described in fig. 3, the outer parts of the laser beam can be viewed as the two beams mentioned above. The interference pattern in the focal plane of the lens is now Gaussian and an optimal measurement of a shift of this pattern can be done with a simple split detector.

Generalizing these ideas, we come to the following rules that should be followed for designing an optimal OBD set-up:

- Light reflected by different points of the cantilever should completely interfere at the detector. This is achieved when the detector plane is the Fourier transform of the cantilever plane. This can be obtained either by using a lens as we did in the first example, or by placing the detector in the far-field region of the cantilever. Essentially this was done when we concluded that $x$ should be larger than $\pi D_{0}^{2} / 4 \lambda$ for an optimal signal-to-noise ratio in our second configuration.

- The total length of the cantilever should be illuminated. This will maximize the differences in phase shift introduced by a displacement of the cantilever for different parts of the beam. This corresponds to the conditions $D=l$ and $D_{0}=l$ in the two cases considered.

- All the available light should be used in order to minimize shot noise.

It has been the aim of this study to compare the fundamental limitations of the OBD with interferometric methods. In practice, other considerations such as available space, beam-pointing stability of the laser, electronic noise, mechanical vibrations, etc. will be equally important design criteria. One of the main problems with 
inteferometric methods is the occurrence of unwanted phase differences between measuring beam and reference beam. This has been greatly reduced by the fiber interferometer of Rugar et al. [9] and the design of Den Boef [7]. In the OBD this is automatically fulfilled. Considered as an interferometer, the interfering beams have essentially the same optical path except in the near-field region of the cantilever.

The reported sensitivities of the various configurations using interferometry and the set-up using optical beam deflection are as follows. For interferometry-based techniques the values are: $1.7 \times 10^{-3} \AA /(\mathrm{Hz})^{1 / 2}$ (homodyne) [5], $8 \times 10^{-4}$ $\AA /(\mathrm{Hz})^{1 / 2}$ (common path) [7], $7 \times 10^{-5} \AA /$ $(\mathrm{Hz})^{1 / 2}$ (polarized differential) [8] and $5.5 \times 10^{-4}$ $\AA /(\mathrm{Hz})^{1 / 2}$ (fiber) [9]. Meyer and Amer [10] reported for the optical beam deflection technique a minimum dectable displacement of $1 \times 10^{-3}$ $\AA /(\mathrm{Hz})^{1 / 2}$. Thus it can be concluded that in practical situations optical beam deflection and interferometry have about the same sensitivity.

\section{Conclusions}

The theoretical analysis presented here shows that a properly designed optical beam deflection configuration is as sensitive as any interferometric method. Because of its simple construction and the absence of a reference beam, optical beam deflection is a very attractive measuring method for an atomic force microscope.

\section{Acknowledgements}

We thank Dr. B. Bölger for a critical reading of this paper. This work was financially supported by the Netherlands Organization for Scientific Research (NWO).

\section{References}

[1] G. Binnig, C.F. Quate and Ch. Gerber, Phys. Rev. Lett. 56 (1986) 930.

[2] T. Göddenhenrich, H. Lemke, U. Hartmann and C. Heiden, J. Vac. Sci. Technol. A 8 (1990) 383.

[3] G. Neubauer, S.R. Cohen, G.M. McClelland, D. Horne and C.M. Mate, Rev. Sci. Instr. 61 (1990) 2296.

[4] Y. Martin and H.K. Wickramasinghe, Appl. Phys. Lett. 50 (1987) 1455.

[5] R. Erlandsson, G.M. McClelland, C.M. Mate and S. Chiang, J. Vac. Sci. Technol. A 6 (1988) 266.

[6] D. Rugar, H.J. Mamin, R. Erlandsson, J.E. Stern and B.D. Terris, Rev. Sci. Instr. 59 (1988) 2337.

[7] A.J. den Boef, PhD Thesis, Eindhoven (1990).

[8] C. Schönenberger and S.F. Alvarado, Rev. Sci. Instr. 60 (1989) 3131.

[9] D. Rugar, H.J. Mamin and P. Guethner, Appl. Phys. Lett. 55 (1989) 2588.

[10] G. Meyer and N.M. Amer, Appl. Phys. Lett. 53 (1988) 2400 .

[11] S. Alexander, L. Hellemans, O. Marti, J. Schneir, V. Elings, P.K. Hansma, M. Longmire and J. Gurley, J. Appl. Phys. 65 (1988) 164.

[12] W.B. Jackson, N.M. Amer, A.C. Boccara and D. Fournier, Appl. Opt. 20 (1981) 1333.

[13] A. Yariv, Quantum Electronics, 3rd ed. (Wiley, New York, 1989) ch. 6. 